\title{
Transient Transfection of the Zoonotic Parasite Babesia microti
}

\author{
Mingming Liu ${ }^{1}$, Shengwei Ji ${ }^{1}$, Mohamed Abdo Rizk ${ }^{1,2}{ }^{\circledR}$, Paul Franck Adjou Moumouni ${ }^{1}$, \\ Eloiza May Galon ${ }^{1}{ }^{1}, \mathrm{Jixu} \mathrm{Li}^{1}{ }^{1}$, Yongchang $\mathrm{Li}^{1}{ }^{1}$, Weiqing Zheng ${ }^{1,3}$, Byamukama Benedicto ${ }^{1}$, \\ Maria Agnes Tumwebaze ${ }^{1}$, Masahito Asada ${ }^{1}$ and Xuenan Xuan ${ }^{1, *}$ \\ 1 National Research Center for Protozoan Diseases, Obihiro University of Agriculture and Veterinary Medicine, \\ Obihiro, Hokkaido 080-8555, Japan; 1mm_2010@hotmail.com (M.L.); jishengwei0903@hotmail.com (S.J.); \\ mohamedabdorizk@gmail.com (M.A.R.); chakirou82@yahoo.fr (P.F.A.M.); eloizagalon@gmail.com (E.M.G.); \\ JixuLi@hotmail.com (J.L.); YongchangLi8762017@outlook.com (Y.L.); zhengweiqing2001@gmail.com (W.Z.); \\ benards.benedicto4@gmail.com (B.B.); tumwebazeaggie@gmail.com (M.A.T.); masada@obihiro.ac.jp (M.A.) \\ 2 Department of Internal Medicine and Infectious Diseases, Faculty of Veterinary Medicine, Mansoura \\ University, Mansoura 35516, Egypt \\ 3 Nanchang Center for Disease Control and Prevention, Nanchang 330038, China \\ * Correspondence: gen@obihiro.ac.jp
}

Received: 7 January 2020; Accepted: 10 February 2020; Published: 10 February 2020

check for updates

\begin{abstract}
The development of genetic manipulation techniques has been reported in many protozoan parasites over the past few years. However, these techniques have not been established for Babesia microti. Here, we report the first successful transient transfection of B. microti. The plasmids containing the firefly luciferase reporter gene were transfected into B. microti by an AMAXA 4D Nucleofection system. Twenty-four-hour synchronization, the $5^{\prime}$-actin promoter, program FA100, and $50 \mu \mathrm{g}$ of plasmid DNA constituted the best conditions for the transient transfection of B. microti. This finding is the first step towards a stable transfection method for B. microti, which may contribute to a better understanding of the biology of the parasite.
\end{abstract}

Keywords: human babesiosis; Babesia microti; transient transfection

\section{Introduction}

The protozoan parasite Babesia microti is the main agent of human babesiosis. This parasite invades and multiplies within red blood cells (RBCs) and infections vary greatly in their presentation depending on the age and immune competency of the host [1]. Severe symptoms are observed in neonates, or in older adults, possibly due to depressed cellular immunity and in the immunocompromised of any age, particularly splenectomized individuals [2]. In patients who were hospitalized with severe B. microti infection, death occurred in $10 \%$ of the cases. Mild disease caused by B. microti usually presents intermittent fever with general malaise and weakness [3].

The difficulties in identifying B. microti virulence factors and developing effective therapies for human babesiosis have been partly attributed to the lack of genetic manipulation tools [4]. The development of these techniques has been reported in bovine and canine Babesia parasites, including B. bovis, B. ovata, B. bigemina, B. gibsoni and B. ovis [5-10]. The application of transfection systems can lead to a better understanding of host-parasite interactions, the mechanisms underlying drug resistance and provides novel information for vaccine development and drug target discovery [11]. For example, transgenic $B$. bovis has been used to analyze gene functions and live vaccines against bovine babesiosis [12-14]. 
In this study, in order to identify the transfection condition for B. microti, the episome plasmids that contain promoters, firefly luciferase reporter, and terminator, were transfected into synchronized B. microti merozoites.

\section{Results}

The schematic diagram of transient transfection described in this study is shown in Figure 1. During the time course of synchronization, parasite viability decreased while the percentage of merozoites significantly increased (Figure 2). Luminescence was shown to require the presence of a parasite and transfection pulse (Figure 3a). Twenty-four-hour synchronized parasites showed higher luciferase activity than the unsynchronized parasites or parasites with 12 and 36-h synchronization (Figure 3b). 5'-actin was the best promoter out of the three candidates tested in this study (Figure 3c). Program FA100 showed higher luciferase activity than other programs (Figure 3d). Fifty micrograms of plasmid DNA was more efficient than 2, 5, and $20 \mu \mathrm{g}$ (Figure 3e). The sequences of B. microti 5'-actin, $5^{\prime}$-ef-1 $\alpha$-subunit, and $5^{\prime}-h s p 70$ promoter were deposited into the GenBank database (accession numbers: MN891916-MN891918). The primers are listed in Table 1.

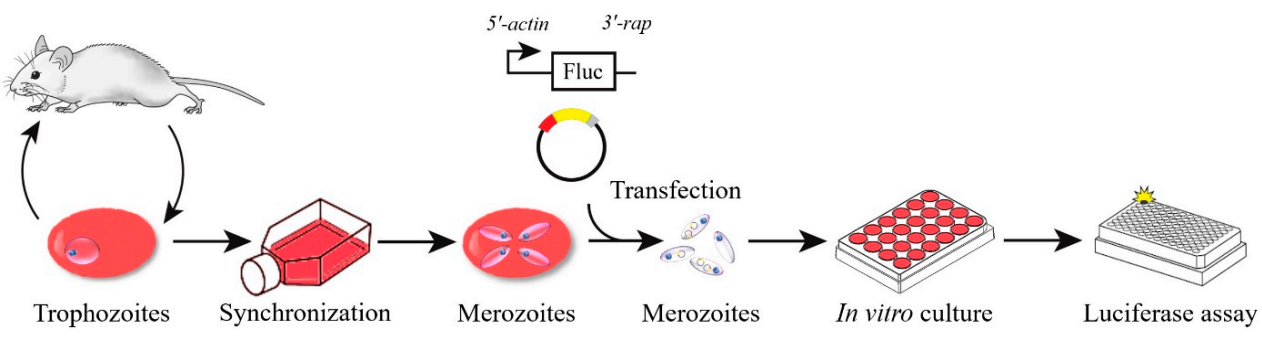

Figure 1. The schematic diagram of transient transfection of B. microti.
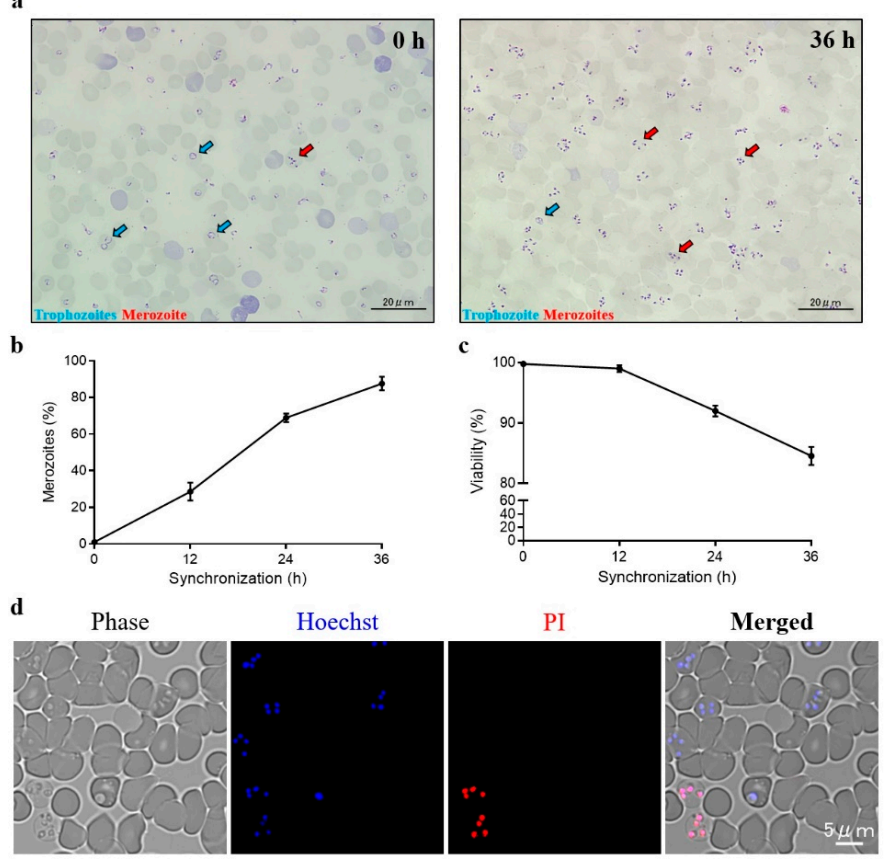

Figure 2. B. microti synchronization. (a) Unsynchronized (left) and 36-h (right) synchronized thin blood smears. (b) The percentages of merozoites were calculated by Giemsa staining. (c) Parasite viability were calculated by Hoechst 33342/Propidium iodide (PI) double staining. (d) Fluorescent microscope images of synchronized B. microti. Parasites were stained by Hoechst 33342/PI double staining at $36 \mathrm{~h}$ post-synchronization. Hoechst 33342 was used to stain the nuclei of both live and dead parasites. PI was used to stain the nuclei of dead parasites. The values are presented as a mean \pm S.D. of three independent experiments. 
a

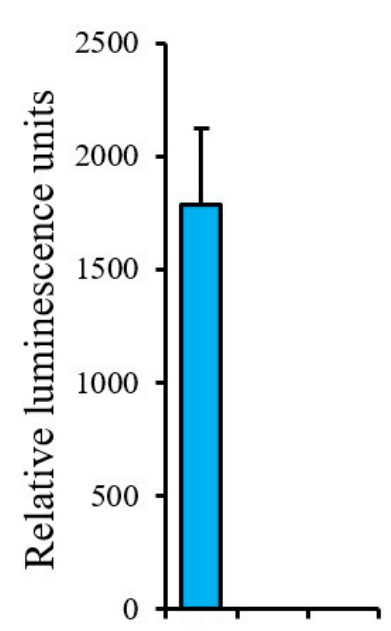

Parasite ++Pulse +-+ b

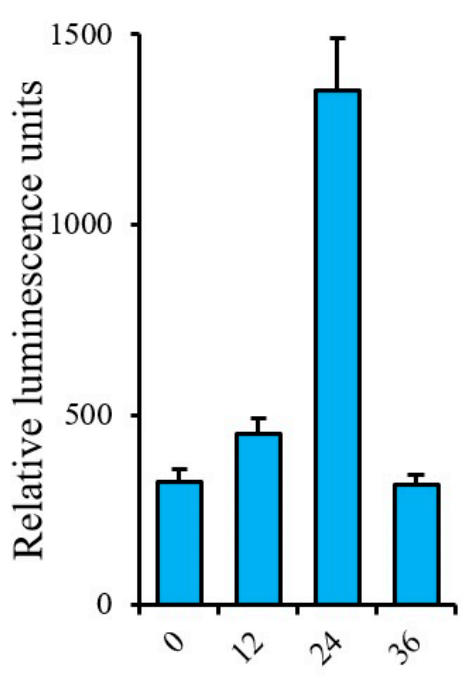

Synchronization (h) c

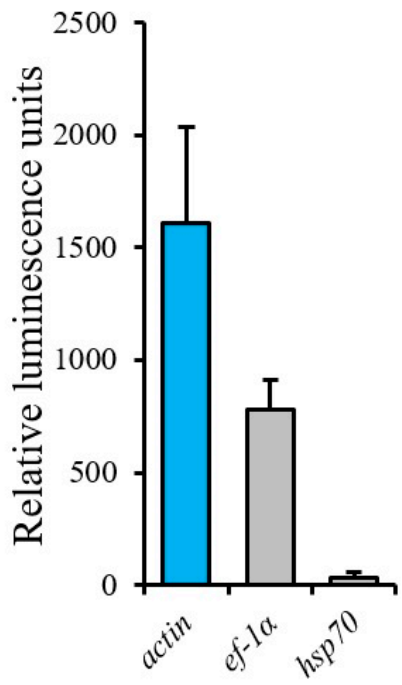

Promoters d

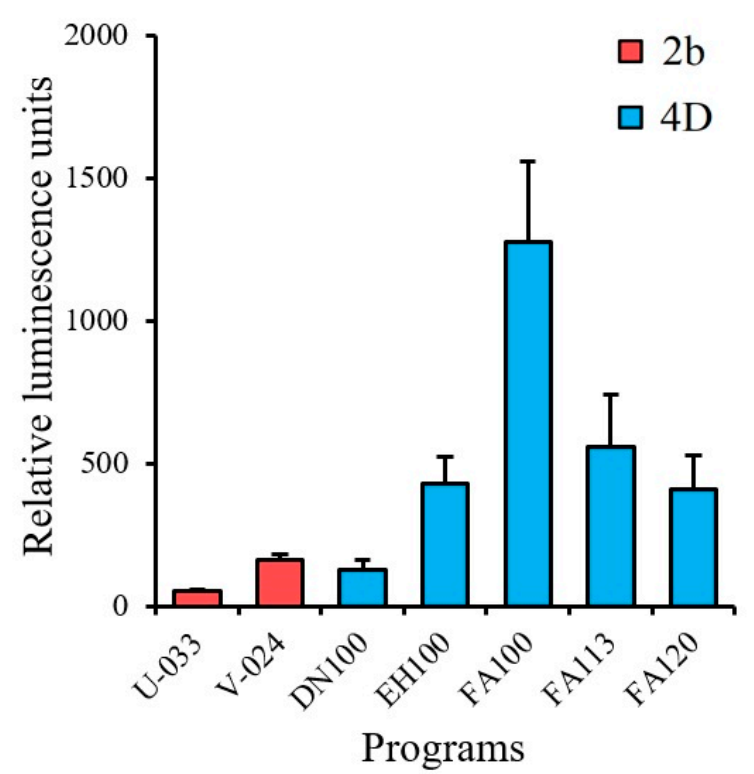

e

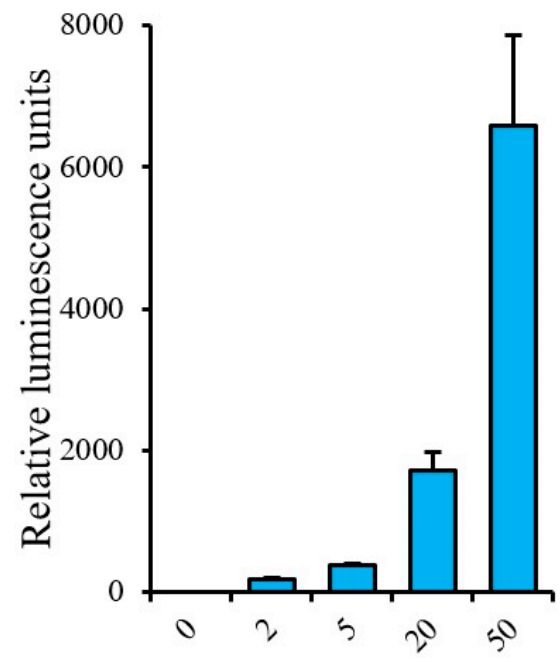

DNA $(\mu \mathrm{g})$

Figure 3. (a) Transient transfection of B. microti. (b) Transfection by various synchronized parasites. (c) Selection of promoters. Actin (5'-actin), ef- $1 \alpha$ (5'-ef- $1 \alpha$-subunit) and $h s p 70$ (5'-hsp70). (d) Selection of programs. 2b (AMAXA Nucleofector 2b using Human T-cell buffer) and 4D (AMAXA 4D Nucleofector using an SF buffer). (e) Transfection by varying amounts of plasmid. Twenty-four-hour synchronized parasites were used in a, c, d, and e. Actin promoter was used in a, b, d, and e. Program FA100 was used in $a, b, c$, and e. Twenty micrograms of plasmid was used in $a, b, c$, and $d$. The values are presented as a mean \pm S.D. of three independent experiments. 
Table 1. List of primers used in this study.

\begin{tabular}{|c|c|c|c|c|}
\hline Element & Primer & Sequence $\left(5^{\prime} \rightarrow 3^{\prime}\right)$ & Size (bp) & Reference \\
\hline \multirow{6}{*}{ Promoter } & Bm 5'-actin (Hind III-F) & GACGGTATCGATAAGCTTATCTTTGTTCCCTTTAGTAT & \multirow{2}{*}{1252} & \multirow{2}{*}{ This study } \\
\hline & Bm 5'-actin (Hind III-R) & GAATTCGATATCAAGCTTTTTATCTAAATTAGAATGTAATT & & \\
\hline & Bm 5'-ef-1 $\alpha$-subunit (Hind III-F) & GACGGTATCGATAAGCTTTCTTTTCTTTTGTGGCGA & \multirow{2}{*}{1152} & \multirow{2}{*}{ This study } \\
\hline & Bm 5'-ef-1 $\alpha$-subunit (Hind III-R) & GAATTCGATATCAAGCTTTTTTCTAACATTCAAGAGGCT & & \\
\hline & Bm 5'-hsp70 (Hind III-F) & GACGGTATCGATAAGCTTTGTTATCATCAGTTACACGCAG & \multirow{2}{*}{1314} & \multirow{2}{*}{ This study } \\
\hline & Bm 5'-hsp70 (Hind III-R) & GAATTCGATATCAAGCTTGTTGGCAGAAATTTCACTCC & & \\
\hline \multirow{2}{*}{ Reporter } & Firefly luciferase (EcoR I-F) & AAGCTTGATATCGAATTCATGGAAGACGCCAAAAACAT & \multirow{2}{*}{1653} & \multirow{2}{*}{ Liu et al. [8] } \\
\hline & Firefly luciferase (EcoR I-R) & CCCGGGCTGCAGGAATTCTTACAATTTGGACTTTCCGCC & & \\
\hline \multirow{2}{*}{ Terminator } & $B b 3^{\prime}-$ rap (Pst I-F) & TTGTAAGAATTCCTGCAGGATGAGATGCGTTTATAATGGC & \multirow[t]{2}{*}{1281} & \multirow[t]{2}{*}{ Liu et al. [15] } \\
\hline & Bb 3'-rap (Pst I-R) & GGATCCCCCGGGCTGCAGCCTACGAACGATATGTCAAAGAG & & \\
\hline
\end{tabular}

\section{Discussion}

In the United States, human babesiosis caused by B. microti is considered an emerging infectious disease. Aside from being transmitted by the hard tick, the majority of the cases are blood transfusiontransmitted babesiosis [16]. Increasing global interconnectivity raises the risk for $B$. microti infections spreading to other countries [17-19]. B. microt $i$ has the smallest nuclear genome among all apicomplexan parasites [20]. Genome-wide phylogenetic analyses indicate that B. microti defines a new clade in the phylum Apicomplexa. Additionally, the difference in both the copy number and organization of multigene families resulted in the phylogeny and life cycle of $B$. microti being significantly distant from those of other Babesia and Theileria parasites [21,22]. Therefore, the established transfection methods for other apicomplexan parasites may not be used for B. microti.

As expected, the common transfection system for bovine Babesia parasites, the AMAXA Nucleofector $2 b$ transfection system (Lonza, Cologne, Germany), was not efficient (Figure 3d). The AMAXA 4D Nucleofector provides a more suitable transfection system for non-bovine Babesia parasites [15]. The transfection system of other parasites was established by this system [23]. However, the optimal transfection program is also different among those parasites, such as FA113 (B. gibsoni) and EH100 (Cryptosporidium parvum), and may not be applicable to B. microti transfection. Overall, the results presented in this study also indicate that $4 \mathrm{D}$ Nucleofection system was more efficient than previous systems for non-bovine Babesia parasites.

Trophozoites were considered to have low transfection efficiency in rodent malaria parasites [24]. A similar observation was noted in this study, as a high percentage of trophozoites (non-synchronization) did not provide good result for $B$. microti transfection (Figure 3b). Unfortunately, the purification of $B$. microti merozoites by using both a Nycodenz density-gradient and a Percoll density-gradient failed (data not shown). As observed for the purification of Plasmodium berghei schizonts, synchronization is needed to increase the percentage of merozoites for transfection [25]. However, over-synchronization can affect the efficiency of transfection (Figure 3b). Because B. microti can only be maintained in a short-term in vitro culture [26], regardless of the red blood cells' $\left(\mathrm{RBCs}^{\prime}\right)$ condition or parasite viability, an over-synchronized parasite may be unsuitable for transfection (Figure 2c,d).

Compared to another human Babesia parasite B. duncani [27], a lack of an in vitro culture system for $B$. microti has severely impacted studies on the parasite, including the search for suitable diagnostic and blood screening markers. Especially for genetic manipulation study, the common drug selection systems used for Babesia parasites, such as human dihydrofolate reductase (hDHFR)/WR99210 and blasticidin-S deaminase (BSD)/bsd selection systems, cannot be used for B. microti [28]. Additionally, B. microti is not sensitive to pyrimethamine, which is the only drug for rodent malaria parasites selection in vivo [29,30]. Overall, stable transfection cannot be fully assessed based on the present study due to challenges such as a lack of a continuous in vitro culture system and unclear selection markers.

In summary, we present the first successful transient transfection of B. microti. This finding is the first step towards a stable transfection method for B. microti, which may contribute to a better 
understanding of the biology of the parasite and paves the way for the development of more effective molecular-based subunit vaccines and the discovery of novel drug targets for controlling human babesiosis. However, further efforts should be put into establishing a continuous in vitro culture system or finding an effective selection marker that can be used in vivo for developing a stable transfection system of B. microti.

\section{Materials and methods}

B. microti Peabody mjr strain (ATCC®PRA-997M) was intraperitoneally administered ( $10^{8}$ parasites) to BALB/c mice. Infected-RBCs (300-350 $\mu \mathrm{L})$ were collected when parasitemia was between $25-30 \%$ and were cultured in vitro in T25 culture flask at $36.5^{\circ} \mathrm{C}$ in an incubator with a humidified atmosphere $\left(5 \% \mathrm{CO}_{2}\right.$ and $\left.5 \% \mathrm{O}_{2}\right)$. The parasites were synchronized in an RPMI-1640 culture medium supplemented with L-glutamine (Gibco, Grand Island, NY, USA), 25 mM HEPES (Sigma, Tokyo, Japan), 0.85 g/L $\mathrm{NaHCO}_{3}$ (Wako, Osaka, Japan), and 20\% fetal bovine serum (FBS) (Biowest, Courtaboeuf, France). Propidium iodide (Sigma) and Hoechst 33342 (Sigma) were used for confirming parasite viability by fluorescence microscopy (Keyence, Osaka, Japan).

Transfection was conducted using a single Nucleocuvette at a final volume of $100 \mu \mathrm{L}$, including $20 \mu \mathrm{g}$ of circular plasmid construct in a $50 \mu \mathrm{L}$ SF buffer and $50 \mu \mathrm{L}$ of synchronized iRBCs $(1.25-1.5$ $\times 10^{8}$ parasites). The plasmid-iRBCs mixtures were transfected using program FA100 of the Amaxa 4D Nucleofection system (Lonza) and immediately transferred into 24-well culture plates with $1 \mathrm{~mL}$ of complete RPMI-1640 culture medium containing 5\% fresh mouse RBCs. The luciferase activity was measured as described previously [8]. Briefly, the transfected iRBCs were ruptured by 10 times volume of $0.8 \% \mathrm{NH}_{4} \mathrm{Cl}$ (Wako) at $24 \mathrm{~h}$ post-transfection, and the parasite pellet was lysed by luciferase assay substrate (Promega, Madison, WI, USA). Finally, the luminescence was measured using a $10 \mathrm{~s}$ integration interval by a GloMax®-Multi Detection System (Promega).

All the procedures were carried out according to ethical guidelines that were approved by Obihiro University of Agriculture and Veterinary Medicine (Permit for animal experiment: 19-120; DNA experiment: 1723-3; Pathogen: 201709-5).

Author Contributions: M.L. and X.X. designed the study. M.L. and S.J. carried out the experiments. M.A.R., J.L., Y.L., W.Z. and M.A. contributed reagents and materials. M.L., P.F.A.M., E.M.G., B.B. and M.A.T. wrote the manuscript. All authors have read and agreed to the published version of the manuscript.

Funding: Mingming Liu is supported by a research grant fellowship from Japan Society for the Promotion of Science (JSPS) for young scientists, Japan (18F18403). This study was supported by a Grants-in-Aid for Scientific Research (18H02336) and JSPS Core-to-Core program, both from the Ministry of Education, Culture, Sports, Science, and Technology of Japan.

Conflicts of Interest: The authors declare no conflict of interest.

\section{References}

1. Parveen, N.; Bhanot, P. Babesia microti-Borrelia Burgdorferi Coinfection. Pathogens 2019, 8, 117. [CrossRef] [PubMed]

2. Bloch, E.M.; Kumar, S.; Krause, P.J. Persistence of Babesia microti Infection in Humans. Pathogens 2019, 8, 102. [CrossRef] [PubMed]

3. Vannier, E.G.; Diuk-Wasser, M.A.; Ben Mamoun, C.; Krause, P.J. Babesiosis. Infect. Dis. Clin. North Am. 2015, 29, 357-370. [CrossRef] [PubMed]

4. Silva, J.C.; Cornillot, E.; McCracken, C.; Usmani-Brown, S.; Dwivedi, A.; Ifeonu, O.O.; Crabtree, J.; Gotia, H.T.; Virji, A.Z.; Reynes, C.; et al. Genome-wide diversity and gene expression profiling of Babesia microti isolates identify polymorphic genes that mediate host-pathogen interactions. Sci. Rep. 2016, 6, 35284. [CrossRef]

5. Hakimi, H.; Ishizaki, T.; Kegawa, Y.; Kaneko, O.; Kawazu, S.-I.; Asada, M. Genome Editing of Babesia bovis Using the CRISPR/Cas9 System. mSphere 2019, 4, e00109-19. [CrossRef]

6. Hakimi, H.; Yamagishi, J.; Kegawa, Y.; Kaneko, O.; Kawazu, S.-I.; Asada, M. Establishment of transient and stable transfection systems for Babesia ovata. Parasites Vectors 2016, 9, 171. [CrossRef] 
7. Silva, M.G.; Knowles, N.P.; Mazuz, M.L.; Cooke, B.M.; Suarez, C.E. Stable transformation of Babesia bigemina and Babesia bovis using a single transfection plasmid. Sci. Rep. 2018, 8, 6096. [CrossRef]

8. Liu, M.; Vudriko, P.; Hakimi, H.; Masatani, T.; Sunaga, F.; Yamagishi, J.; Xuan, X.; Asada, M.; Cao, S.; Moumouni, P.F.A.; et al. Transient transfection of intraerythrocytic Babesia gibsoni using elongation factor-1 alpha promoter. Mol. Biochem. Parasitol. 2017, 216, 56-59. [CrossRef]

9. Rosa, C.; Asada, M.; Hakimi, H.; Domingos, A.; Pimentel, M.; Antunes, S. Transient transfection of Babesia ovis using heterologous promoters. Ticks Tick-borne Dis. 2019, 10, 101279. [CrossRef]

10. Liu, M.; Moumouni, P.F.A.; Cao, S.; Asada, M.; Wang, G.; Gao, Y.; Guo, H.; Li, J.; Vudriko, P.; Efstratiou, A.; et al. Identification and characterization of interchangeable cross-species functional promoters between Babesia gibsoni and Babesia bovis. Ticks Tick-borne Dis. 2018, 9, 330-333. [CrossRef]

11. Suarez, C.E.; Noh, S. Emerging perspectives in the research of bovine babesiosis and anaplasmosis. VeterParasitol. 2011, 180, 109-125. [CrossRef] [PubMed]

12. Alzan, H.F.; Cooke, B.M.; Suarez, C.E. Transgenic Babesia bovis lacking 6-Cys sexual-stage genes as the foundation for non-transmissible live vaccines against bovine babesiosis. Ticks Tick-borne Dis. 2019, 10, 722-728. [CrossRef] [PubMed]

13. Gallego-Lopez, G.M.; Lau, A.O.; O'Connor, R.M.; Ueti, M.W.; Cooke, B.M.; Laughery, J.M.; Graça, T.; Madsen-Bouterse, S.A.; Oldiges, D.P.; Allred, D.R.; et al. Up-regulated expression of spherical body protein 2 truncated copy 11 in Babesia bovis is associated with reduced cytoadhesion to vascular endothelial cells. Int. J. Parasitol. 2019, 49, 127-137. [CrossRef] [PubMed]

14. Hussein, H.E.; Bastos, R.G.; Schneider, D.A.; Johnson, W.C.; Adham, F.K.; Davis, W.C.; Laughery, J.M.; Herndon, D.R.; Alzan, H.F.; Ueti, M.W.; et al. The Babesia bovis hap2 gene is not required for blood stage replication, but expressed upon in vitro sexual stage induction. PLOS Neglected Trop. Dis. 2017, 11, e0005965. [CrossRef]

15. Liu, M.; Moumouni, P.F.A.; Asada, M.; Hakimi, H.; Masatani, T.; Vudriko, P.; Lee, S.-H.; Kawazu, S.-I.; Yamagishi, J.; Xuan, X. Establishment of a stable transfection system for genetic manipulation of Babesia gibsoni. Parasites Vectors 2018, 11, 260. [CrossRef]

16. Moritz, E.D.; Winton, C.S.; Tonnetti, L.; Townsend, R.L.; Berardi, V.P.; Hewins, M.E.; Weeks, K.E.; Dodd, R.Y.; Stramer, S.L. Screening for Babesia microti in the U.S. blood supply. N. Engl. J. Med. 2016, 375, 2236-2245. [CrossRef]

17. Lim, P.L.; Chavatte, J.M.; Vasoo, S.; Yang, J. Imported Human Babesiosis, Singapore, 2018. Emerg. Infect. Dis. 2020, 26. [CrossRef]

18. Zamoto-Niikura, A.; Morikawa, S.; Hanaki, K.-I.; Holman, P.J.; Ishihara, C. Ixodes persulcatus Ticks as Vectors for the Babesia microti U.S. Lineage in Japan. Appl. Environ. Microbiol. 2016, 82, 6624-6632. [CrossRef]

19. Gabrielli, S.; Totino, V.; Macchioni, F.; Zuñiga, F.; Rojas, P.; Lara, Y.; Roselli, M.; Bartoloni, A.; Cancrini, G. Human Babesiosis, Bolivia, 2013. Emerg. Infect. Dis. 2016, 22, 1445-1447. [CrossRef]

20. Cornillot, E.; Hadj-Kaddour, K.; Dassouli, A.; Noel, B.; Ranwez, V.; Vacherie, B.; Augagneur, Y.; Brès, V.; Duclos, A.; Randazzo, S.; et al. Sequencing of the smallest Apicomplexan genome from the human pathogen Babesia microti. Nucleic Acids Res. 2012, 40, 9102-9114. [CrossRef]

21. Jalovecka, M.; Hajdusek, O.; Sojka, D.; Kopacek, P.; Malandrin, L. The Complexity of Piroplasms Life Cycles. Front. Microbiol. 2018, 8, 248. [CrossRef] [PubMed]

22. Schreeg, M.E.; Marr, H.S.; Tarigo, J.L.; Cohn, L.A.; Bird, D.M.; Scholl, E.H.; Levy, M.G.; Wiegmann, B.M.; Birkenheuer, A.J. Mitochondrial genome sequences and structures aid in the resolution of Piroplasmida phylogeny. PLoS ONE 2016, 11, e0165702. [CrossRef] [PubMed]

23. Vinayak, S.; Pawlowic, M.C.; Sateriale, A.; Brooks, C.F.; Studstill, C.J.; Bar-Peled, Y.; Cipriano, M.J.; Striepen, B. Genetic modification of the diarrhoeal pathogen Cryptosporidium parvum. Nature 2015, 523, 477-480. [CrossRef] [PubMed]

24. Janse, C.J.; Franke-Fayard, B.; Mair, G.R.; Ramesar, J.; Thiel, C.; Engelmann, S.; Matuschewski, K.; Van Gemert, G.J.; Sauerwein, R.W.; Waters, A.P.; et al. High efficiency transfection of Plasmodium berghei facilitates novel selection procedures. Mol. Biochem. Parasitol. 2006, 145, 60-70. [CrossRef]

25. Janse, C.J.; Ramesar, J.; Waters, A.P. High-efficiency transfection and drug selection of genetically transformed blood stages of the rodent malaria parasite Plasmodium berghei. Nat. Protoc. 2006, 1, 346-356. [CrossRef] [PubMed] 
26. Shikano, S.; Nakada, K.; Hashiguchi, R.; Shimada, T.; Ono, K. A Short Term in Vitro Cultivation of Babesia rodhaini and Babesia microti. J. Veter-Med. Sci. 1995, 57, 955-957. [CrossRef]

27. Abraham, A.; Brasov, I.; Thekkiniath, J.; Kilian, N.; Lawres, L.; Gao, R.; Debus, K.; He, L.; Yu, X.; Zhu, G.; et al. Establishment of a continuous in vitro culture of Babesia duncani in human erythrocytes reveals unusually high tolerance to recommended therapies. J. Boil. Chem. 2018, 293, 19974-19981. [CrossRef] [PubMed]

28. Asada, M.; Yahata, K.; Hakimi, H.; Yokoyama, N.; Igarashi, I.; Kaneko, O.; Suarez, C.E.; Kawazu, S.-I. Transfection of Babesia bovis by Double Selection with WR99210 and Blasticidin-S and Its Application for Functional Analysis of Thioredoxin Peroxidase-1. PLoS ONE 2015, 10, e0125993. [CrossRef] [PubMed]

29. Yao, J.-M.; Zhang, H.-B.; Liu, C.-S.; Tao, Y.; Yin, M. Inhibitory effects of 19 antiprotozoal drugs and antibiotics on Babesia microti infection in BALB/c mice. J. Infect. Dev. Ctries. 2015, 9, 1004-1010. [CrossRef]

30. Manzoni, G.; Briquet, S.; Risco-Castillo, V.; Gaultier, C.; Topçu, S.; Ivănescu, M.L.; Franetich, J.-F.; Hoareau-Coudert, B.; Mazier, M.; Silvie, O. A rapid and robust selection procedure for generating drug-selectable marker-free recombinant malaria parasites. Sci. Rep. 2014, 4, 4760. [CrossRef]

(C) 2020 by the authors. Licensee MDPI, Basel, Switzerland. This article is an open access article distributed under the terms and conditions of the Creative Commons Attribution (CC BY) license (http://creativecommons.org/licenses/by/4.0/). 